\title{
O ENSINO DA PSICOLOGIA NA GRADUAÇÃO DE FONOAUDIOLOGIA
}

THE TEACHING OF PSYCHOLOGY ON THE SPEECH AND LANGUAGE THERAPY GRADUATION

\author{
Patricia L. dos Santos ${ }^{1}$, Maria de Lourdes V. Rodrigues ${ }^{2}$
}

\begin{abstract}
${ }^{1}$ Docente. Departamento de Neurologia, Psiquiatria e Psicologia Médica. ${ }^{2}$ Docente. Departamento de Oftalmologia, Otorrinilaringologia e Cirurgia de Cabeça e Pescoço. Membro do Grupo de Consultores do Centro de Apoio Educacional e Psicológico. Faculdade de Medicina de Ribeirão Preto - USP.

Correspondência: Patricia Leila dos Santos. Rua Tenente Catão Roxo, 2650, Vila Monte Alegre CEP 14051-140 Ribeirão Preto, SP, e-mail: plsantos@fmrp.usp.br
\end{abstract}

Santos PL, Rodrigues MLV. O ensino da Psicologia na graduação de Fonoaudiologia. Medicina (Ribeirão Preto) 2007, 40 (1): 78-81.

RESUMO: As Diretrizes Curriculares Nacionais do Curso de Graduação em Fonoaudiologia prevê, entre os temas essenciais para a formação deste profissional, conteúdos da área da Psicologia. Esta assume um papel importante na formação do profissional da fala, possibilitando a compreensão dos fenômenos comportamentais, psíquicos e também das relações entre os seres humanos e seus contextos de desenvolvimento, ampliando, assim, a compreensão dos processos de comunicação saudáveis ou patológicos e complementando sua formação clínica a partir de conhecimentos sobre técnicas de manejo dos pacientes. Entretanto, acompanhando a variedade dos currículos dos cursos de Fonoaudiologia, também é bastante diverso o número e o conteúdo das disciplinas de Psicologia ministradas nos cursos. Enquanto docentes de cursos de graduação em especialidades diversa da nossa, e como administradora do Departamento, temos nos preocupado em elaborar programas que articulem os saberes e atendam à necessidade do profissional em formação, preparando-o para exercer suas atividades de forma autônoma. Com a recente implantação do curso de Fonoaudiologia na Faculdade de Medicina de Ribeirão Preto da Universidade de São Paulo, estamos realizando estudos visando aprimorar os programas das disciplinas propostas, especialmente na área de Psicologia, de modo a atender às necessidades profissionais do fonoaudiólogo e às demandas do mercado de trabalho.

Descritores: Psicologia. Ensino. Fonoaudiologia. Graduação. Educação em Saúde.

A Fonoaudiologia é uma profissão relativamente nova no Brasil e em várias partes do mundo, sendo que em muitos países os fonoaudiólogos ainda lutam pelo reconhecimento e autonomia da profissão ${ }^{1,2}$.

No Brasil, no início da década de 60 , foi criado o primeiro curso de Fonoaudiologia, junto à Universidade de São Paulo, sendo que apenas em 1981, a profissão foi reconhecida como ocupação oficial. Hoje são 111 cursos de graduação em todo o país, além de cursos de Mestrado e Doutorado, e quatro áreas de especialização reconhecidas pelo Conselho Federal de Fonoaudiologia: audição, linguagem, voz e motricidade oral $^{3,4}$.

Os cursos têm uma variedade de características, sendo que alguns enfatizam tópicos relacionados a aspectos orgânicos, outros privilegiam temas psicoló- 
gicos, educacionais e sociais, embora todos eles preparem profissionais para trabalhar tanto em audiologia quanto na terapia da fala e linguagem. Normalmente os currículos oferecem anatomia, fisiologia, lingüística, psicologia, física acústica, sociologia, educação, entre outras disciplinas ${ }^{3}$.

Além da variedade de tópicos, as disciplinas diferem de um curso para outro, especialmente quanto a conteúdos, apesar da orientação das Diretrizes Curriculares Nacionais (DCN) do Curso de Graduação em Fonoaudiologia.

De acordo com as DCN, os cursos devem formar um profissional generalista, humanista, crítico e reflexivo. Assim, o fonoaudiólogo deve estar preparado para desenvolver ações de prevenção, promoção, proteção e reabilitação da saúde; trabalhar de forma integrada e contínua com as demais instâncias do sistema de saúde; tomar decisões, possuindo competências e habilidades para avaliar, sistematizar e decidir as condutas mais adequadas, baseadas em evidências científicas; estar preparado para comunicar-se adequadamente com outros profissionais de saúde e com o público em geral, bem como para o trabalho em equipe multiprofissional e para administrar e gerenciar recursos humanos, físicos e financeiros, além de estar compromissado com a sua própria educação e com a formação das futuras gerações de profissionais ${ }^{5}$.

Ainda, as DCN orientam que, além de qualificar o profissional para compreender, analisar e atuar de acordo com os sistemas teóricos e conceituais envolvidos no campo fonoaudiológico (motricidade oral, voz, fala, linguagem oral e escrita e da audição, e os métodos clínicos utilizados para prevenir, avaliar, diagnosticar e tratar os distúrbios da linguagem - oral e escrita - audição, voz e sistema sensório motor oral), prepare o fonoaudiólogo para compreender os processos envolvidos na constituição do ser humano, nas relações sociais, no psiquismo, na linguagem, na aprendizagem, bem como o estudo destes processos como condição para a compreensão da gênese e da evolução das alterações fonoaudiológicas ${ }^{5}$.

Os conteúdos essenciais para o Curso de Graduação em Fonoaudiologia devem estar relacionados com todo o processo saúde-doença do cidadão, da família e da comunidade, integrado à realidade epidemiológica e profissional, proporcionando a integralidade das ações do cuidar em fonoaudiologia, englobando conteúdos das ciências biológicas e da saúde (bases moleculares e celulares dos processos normais e alte- rados, da estrutura e função dos tecidos, órgãos, sistemas e aparelhos), das ciências sociais e humanas (determinantes sociais, culturais, econômicos, comportamentais, psicológicos, ecológicos, éticos e legais, lingüísticos e educacionais da saúde) e ciências fonoaudiológicas (audição, linguagem oral e escrita, voz, fala, fluência e sistema miofuncional orofacial e cervical $)^{5}$.

Preconiza-se que o fonoaudiólogo seja capaz de compreender os diferentes processos que afetam o ser humano. Assim, as diretrizes educacionais contemplam a necessidade de uma formação voltada para a integralidade das ações do cuidar em Fonoaudiologia, ou seja, que não se restrinja apenas ao tratamento dos distúrbios da fala e linguagem, ignorando o contexto social e as necessidades individuais dos pacientes, e que realize ações de prevenção e promoção de saúde.

Neste contexto, a Psicologia assume um papel importante na formação do profissional da fala, possibilitando a compreensão dos fenômenos comportamentais, psíquicos e também das relações entre os seres humanos e seus contextos de desenvolvimento, ampliando, assim, a compreensão dos processos de comunicação saudáveis ou patológicos e complementando sua formação clínica a partir de conhecimentos sobre técnicas de manejo dos pacientes. Além disso, através de técnicas da Psicologia pode-se ajudar os profissionais a desenvolverem diferentes habilidades sociais e de trabalho (inclusive de comunicação e liderança) necessárias para o bom desenvolvimento das suas atividades.

Entretanto, acompanhando a variedade dos currículos dos cursos de Fonoaudiologia, também é bastante diverso o número e o conteúdo das disciplinas de Psicologia ministradas nos cursos ${ }^{6}$.

Tomando, como exemplo, o currículo de 12 dos 31 cursos de Fonoaudiologia existentes no Estado de São Paulo, encontramos um elenco diverso de disciplinas ligadas à área de Psicologia, que podem ser agrupadas nos seguintes temas: Psicologia do desenvolvimento, Psicologia da aprendizagem, Introdução à Psicologia, Introdução à Psicanálise, Aconselhamento familiar, Psicologia aplicada à Fonoaudiologia (relação terapêutica, pacientes especiais), Psicologia geral, Psicolingüística, Relações interpessoais e dinâmica de grupos, Psicomotricidade, Psicopatologia, Psicologia do excepcional, Psicopedagogia, Distúrbios da aprendizagem e Psicodiagnóstico. 
Os diferentes conteúdos contribuem para que o fonoaudiólogo possa conhecer as principais características dos indivíduos em diferentes etapas da vida, as psicopatologias associadas aos distúrbios da comunicação, os diferentes aspectos envolvidos no fenômeno do relacionamento humano, bem como estratégias de intervenção, favorecendo tanto a avaliação quanto o planejamento de ações em Fonoaudiologia.

Quanto à literatura sobre o ensino de Fonoaudiologia, ela é bastante escassa. Num levantamento realizado em várias bases de dados, encontramos apenas um artigo ${ }^{7}$ que relata brevemente as alterações ocorridas no sistema educacional japonês para os terapeutas de fala após a apresentação das Diretrizes Educacionais propostas pela International Association of Logopedics and Phoniatrics (IALP).

Contudo, faz-se necessário olhar para a formação destes mesmos profissionais, especialmente no momento atual, em que cada vez mais os profissionais de saúde têm observado a necessidade de conhecimentos multidisciplinares para uma melhor avaliação, planejamento e encaminhamento dos pacientes atendidos.

Particularmente, enquanto docentes em cursos de formação de especialistas de áreas diversas das nossas especialidades (Psicologia e Oftalmologia), nos vimos às voltas com o dilema: em que basear nossos programas de disciplinas? Podemos tomar dois caminhos distintos. O primeiro, talvez mais fácil para nós, montando os programas a partir do nosso referencial, oferecendo aos alunos de diferentes cursos de graduação conteúdos que, do ponto de vista de nossas especialidades e experiências profissionais, consideramos importantes na formação dos outros profissionais. $\mathrm{O}$ segundo nos obriga a entrar em contato com os conteúdos e necessidades oriundas de outras áreas de conhecimento, muitas vezes desconhecidas para nós, o que torna o trabalho mais árduo: elaborar programas que incluem conteúdos que venham a atender às necessidades próprias dos profissionais de outras especialidades.

Podemos pensar ainda, num terceiro caminho, que articule os dois processos anteriores, estabelecendo o diálogo com os outros profissionais, mostrando que contribuições nossas especialidades poderiam oferecer e ouvindo as necessidades dos profissionais de outras áreas sobre suas dificuldades e necessidades de conhecimento e, então, elaborar os programas con- juntamente, pautados nas contribuições e referenciais das áreas de conhecimento envolvidas.

Nossa preocupação tem sido exatamente esta: elaborar programas que articulem os saberes e atendam à necessidade do profissional em formação, preparando-o para exercer suas atividades de forma autônoma. É importante ter claro que, embora alguns temas sejam comuns, cada área tem suas especificidades e demandas quanto a conhecimentos multidisciplinares.

Em algumas áreas da saúde, como a Medicina, a Odontologia e a Enfermagem, o estudo sobre ensino e metodologias educacionais têm ganhado espaço e têm sido realizados trabalhos que buscam conhecer as necessidades específicas de cada profissional, as deficiências existentes na formação e que apresentam propostas que atendem tanto aos objetivos da formação quanto às necessidades da população atendida por eles. Estes estudos destacam as contribuições que diferentes especialidades podem ter na formação dos profissionais de saúde ${ }^{8,9,10}$.

Os atuais currículos na área da saúde trabalham com um modelo de organização no qual espera-se que aconteça primeiro a aprendizagem de referenciais teóricos que, mais tarde serão aplicados à prática. Entretanto, é imprescindível compreender as circunstâncias nas quais as aprendizagens ocorrem, para que se possa pensar em modificações ou inovações curriculares a partir da estreita relação teoriaprática $^{11}$.

Em nosso contexto de trabalho, tendo em vista a recente implantação do curso de Fonoaudiologia na Faculdade de Medicina de Ribeirão Preto da Universidade de São Paulo, com início em 2003, estamos realizando estudos visando aprimorar os programas das disciplinas propostas, especialmente na área de Psicologia, de modo a atender às necessidades profissionais do fonoaudiólogo que será formado, atendendo também às demandas do mercado de trabalho.

Acreditamos, concordando com Fagundes e Burnham ${ }^{12}$, que pensar em currículos mais sensíveis às necessidades do trabalho, às demandas localizadas, significa o desenvolvimento de uma capacidade de escuta às práticas curriculares nos espaços em que elas ocorrem e a outros espaços sociais e profissionais em que se aprende e se trabalha a saúde, favorecendo a questionamentos e às demandas do processo de formação. 
Santos PL, Rodrigues MLV. The teaching of psychology on the speech and language therapy graduation. Medicina (Ribeirão Preto) 2007, 40 (1): 78-81.

ABSTRACT: The National Curricular Directives of the Undergraduate Speech Therapy Course consider contents in the area of Psychology to be among the essential topics for the training of these professionals. Psychology plays an important role in the training of speech professionals, permitting the understanding of behavioral and psychic phenomena and of the relations among human beings and their developmental contexts. Thus, it expands the understanding of healthy or pathological communication processes and complements the clinical training of these professionals based on knowledge about techniques of patient management. However, a survey of the curricular variety of Speech Therapy courses reveals that the number and content of Psychology disciplines taught in these courses are quite diverse. As teachers of undergraduate courses in a specialty different from ours and as administrator of the Department, we have been concerned about elaborating programs that will articulate knowledge and will satisfy the needs of training professionals, preparing them to carry out their activities in an autonomous manner. With the recent implantation of the Speech Therapy course at the Faculty of Medicine of Ribeirão Preto, University of São Paulo, we are conducting studies aiming at the refinement of the disciplines proposed, especially in the area of Psychology, in order to satisfy the professional needs of speech therapists and the demands of the work market.

Keywords: Psychology, Teaching. Speech Therapy, Graduation. Health Education.

\section{REFERÊNCIAS}

1 - Nilipour R. Emerging issues in speech therapy in Iran. Folia Phoniatr Logop 2002; 54(2): 65-8.

2 - Korenbrot F, Hertzano T, Ben Aroya, A. Emerging issues in Israel: commentaries in a global context. Folia Phoniatr Logop 2002; 54(2): 72-4.

3 - Ferreira, LP. Speech therapy in Brazil: Forty years of existence, two decades of recognition. Folia Phoniatr Logop 2002; 54(2): 103-5.

4 - Instituto Nacional de Estudos e Pesquisas em Educação INEP. 2006. Cadastro de Educação Superior. Disponível em http://www.educacaosuperior.inep.gov.br (acessado em 2 de junho de 2006).

5 - Ministério da Educação. Conselho Nacional de Educação. 2002. Diretrizes Curriculares Nacionais dos Cursos de Graduação em Fisioterapia, Fonoaudiologia e terapia ocupacional. Disponível em http://www.mec.gov.br/sesu (acessado em 2 de junho de 2006).

6 - Conselho Regional de Fonoaudiologia. 2ª . Região. 2006. Faculdades. Disponível em http://www.fonosp.org.br (acessado em 25 de maio de 2006).
7 - litaka K. Japanese speech therapy (logaoedics) education after the presentation of the education guidelines in the 1995 IALP general assembly. Folia Phoniatr Logop 2002; 54(2): 758.

8 - Kent G. The way we teach Psychology to Dental Students. Med Teach 1985; 7(3/4):289-95.

9 - Ballester DA, Filippon AP, Braga C, Andreoli SB. The general practitioner and mental health problems: challenges and strategies for medical education. Sao Paulo Med J 2005, 123(2): 72-6.

10 - Patrocínio LG, Silveira GC, Patrocínio TG, Patrocínio JA. Avaliação de necessidades para um currículo de otorrinolaringologia na graduação. Rev Bras Otorrinolaringol 2002, 68(1): 107-11.

11 - Toralles-Pereira ML. Notas sobre educação na transição para um novo paradigma. Interface (Botucatu) 1997, 1(1): 51-68.

12 - Fagundes NC, Burnham TF. Discutindo a relação entre espaço e aprendizagem na formação de profissionais de saúde. Interface (Botucatu) 2005; 9(16): 105-14.

Recebido em 18/08/2006

Aprovado em 24/11/2006 